\title{
Discordance in diagnosis of osteoporosis using spine and hip bone densitometry
}

\author{
Alireza Moayyeri*1,2, Akbar Soltani ${ }^{1,2}$, Nasibeh Khaleghnejad Tabari ${ }^{1}$, \\ Mohsen Sadatsafavi ${ }^{2}$, Arash Hossein-neghad ${ }^{1}$ and Bagher Larijani ${ }^{1}$
}

\begin{abstract}
Address: ${ }^{1}$ Endocrinology \& Metabolism Research Centre, Shariati Hospital, Tehran University of Medical Sciences, Tehran, Iran and ${ }^{2}$ Research Development Center, Evidence Based Medicine Working Team, Shariati hospital, Tehran University of Medical Sciences, Tehran, Iran

Email: Alireza Moayyeri* - moayyeri@doctor.com; Akbar Soltani - soltania@sina.tums.ac.ir;

Nasibeh Khaleghnejad Tabari - emrc@sina.tums.ac.ir; Mohsen Sadatsafavi - safavi@ddrcir.org; Arash Hossein-neghad - emrc@sina.tums.ac.ir; Bagher Larijani - emrc@sina.tums.ac.ir

* Corresponding author
\end{abstract}

Published: II March 2005

BMC Endocrine Disorders 2005, 5:3 doi:10.1/86/1472-6823-5-3

This article is available from: http://www.biomedcentral.com//472-6823/5/3

(c) 2005 Moayyeri et al; licensee BioMed Central Ltd.

This is an Open Access article distributed under the terms of the Creative Commons Attribution License (http://creativecommons.org/licenses/by/2.0), which permits unrestricted use, distribution, and reproduction in any medium, provided the original work is properly cited.
Received: 28 October 2004

Accepted: II March 2005

\begin{abstract}
Background: Diagnostic discordance for osteoporosis is the observation that the T-score of an individual patient varies from one key measurement site to another, falling into two different diagnostic categories identified by the World Health Organization (WHO) classification system. This study was conducted to evaluate the presence and risk factors for this phenomenon in a large sample of Iranian population.
\end{abstract}

Methods: Demographic data, anthropometric measurements, and risk factors for osteoporosis were derived from a database on $\mathbf{4 2 2 9}$ patients referred to a community-based outpatient osteoporosis testing center from 2000 to 2003. Dual-energy X-ray absorptiometry (DXA) was performed on LI-L4 lumbar spine and total hip for all cases. Minor discordance was defined as present when the difference between two sites was no more than one WHO diagnostic class. Major discordance was present when one site is osteoporotic and the other is normal. Subjects with incomplete data were excluded.

Results: In 4I 88 participants (3848 female, mean age $53.4 \pm$ I I.8 years), major discordance, minor discordance, and concordance of T-scores were seen in $2.7 \%, 38.9 \%$ and $58.3 \%$, respectively. In multivariate logistic regression analysis, older age, menopause, obesity, and belated menopause were recognized as risk factors and hormone replacement therapy as a protective factor against $\mathrm{T}$ score discordance.

Conclusion: The high prevalence of T-score discordance may lead to problems in interpretation of the densitometry results for some patients. This phenomenon should be regarded as a real and prevalent finding and physicians should develop a particular strategy approaching to these patients.

\section{Background}

Osteoporosis is defined as a systemic skeletal disease characterized by low bone mass and micro-architectural dete- rioration of bone tissue, with a consequent increase in bone fragility and susceptibility to fracture [1,2]. This definition indicates that measurement of bone mineral 
density (BMD) is a central component to diagnosis of the disease [3].

'T score' is a statistical definition which indicates the difference between patient's BMD and mean bone density of normal population in the age of $20-30$ (reference population) [3]. This value shows the difference in terms of standard deviations. According to the World Health Organization (WHO) classification system, T scores under the value of -2.5 are considered as osteoporosis and between -1 and -2.5 as osteopenia. These figures are usually calculated separately for two different sites of lumbar spine and total hip.

Discordance in diagnosis of osteoporosis is defined as presence of different categories of T scores (osteoporosis, osteopenia, and normal) in two skeletal sites of an individual patient [4]. This phenomenon has been divided into two groups: major and minor [5]. Minor discordance happens when the different diagnostic classes are adjacent; i.e., patient is diagnosed as osteoporotic in one site and osteopenic in the other site, or, osteopenic in one site and normal in the other site. If the diagnosis is osteoporosis in one site and the other site is in the normal range, the discordance falls into the major class.

Actually, one of the reasons for measuring BMD in several sites is the presence of discordance, which can affect the diagnosis and therapeutic plan in an individual person. Various studies have analyzed the prevalence and impact of T-score discordance on different aspects of management of osteoporosis [5-9]. However, most of these studies did not evaluate risk factors for this phenomenon.

Given this background and concerning the need for the estimation of the impact of this phenomenon in our country, we aimed to evaluate the presence and risk factors for T-score discordance in a large sample of Iranian population.

\section{Methods}

Participants in this study were 4229 persons who underwent bone densitometry in outpatient clinic of Endocrinology \& Metabolism Research Center in Tehran from 2000 to 2003. A considerable proportion of these cases were healthy post-menopausal women referred by clinicians for densitometric evaluations. All study participants signed the informed consent for any scientific approach to their medical registered data. Our Institutional Review Board approved this study.

A standardized questionnaire was filled before densitometry for all participants. Demographic data (including age and sex) as well as other known or suspicious risk factors for osteoporosis (including menopause, age at meno- pause, age at menarche, history of osteoporotic fractures, drugs, and smoking) were collected. All participants had their standing height measured using a stadiometer to the nearest $0.5 \mathrm{~cm}$. Weight was measured on a standard weighting scale with a precision of $0.5 \mathrm{~kg}$. Body mass index (BMI) was calculated as weight $(\mathrm{kg})$ divided by height $(\mathrm{m})$ squared. All the BMD measurements were done for diagnostic purposes and none of the participants were on the treatment with bone active agents (hormone replacement therapy was not considered a bone active agent).

BMD was measured at the lumbar spine and total hip with dual X-ray absorptiometry (DXA) using a Lunar DPXMD densitometer (Lunar 7164, GE, Madison, WI) by a trained operator according to the manufacturer's instruction. The instrument was calibrated weekly by using appropriate phantoms. Precision error for BMD measurements was 1$1.5 \%$ in the lumbar and $2-3 \%$ in the femoral regions. The device normative data of US population for spine BMD and NHANES III study for femur BMD were used as reference values.

All the data gained from densitometry and questionnaires were entered into a comprehensive relational database. The participants with incomplete data were excluded from the study. To compare presence of various risk factors in participants with and without T-score discordance, chisquare test and independent sample t-test were used firstly. Potential risk factors were entered to a multivariate binary logistic regression analysis and the resulted odds ratios with $95 \%$ confidence intervals were reported. $P$ values less than 0.05 were taken to indicate statistical significance. Statistical analyses were performed using Stata Statistical Package, version 8.0 (Stata Corporation, College Station, Tx).

\section{Results}

In sum, 4188 persons were enrolled in the study. Characteristics of all participants are summarized in Table 1 . The main reasons of referral for BMD measurement were menopause in $49 \%$, old age in $16 \%$, glucocorticoid use in $9 \%$, history of low energy fractures in $1.5 \%$, and other reasons (such as metabolic disorders, rheumatoid arthritis, positive family history, leanness, and transplantation) in $4.5 \%$ of participants. In $20 \%$ of participants, no major risk factor was identified as the referral reason.

Totally, 518 participants were diagnosed in osteoporotic range in hip area and 1036 participants in the lumbar area. T-score classifications are presented in Table 2. Major discordance was observed in BMD results of 115 (2.7\%) participants. Minor discordance was observed in 1631 (38.9\%) participants and T-score categories of two 
Table I: Characteristics of the study population*

\begin{tabular}{ccc}
\hline & Male participants $(\mathbf{n}=\mathbf{3 4 0})$ & Female participants $(\mathbf{n}=\mathbf{3 8 4 8})$ \\
\hline Age (years) & $49.7(16.3)$ & $53.8(11.2)$ \\
Weight (kilograms) & $68.5(13.1)$ & $67.1(11.9)$ \\
Height (centimeters) & $168.5(7.7)$ & $156.1(6.1)$ \\
Body Mass Index (kg/cm $)$ & $24.1(4.2)$ & $27.6(4.7)$ \\
History of osteoporotic fracture & $8(2.4)$ & $47(1.2)$ \\
Smoking & $35(10.3)$ & $94(2.4)$ \\
Corticosteroid use & $89(26.2)$ & $298(7.7)$ \\
Hormone Replacement Therapy & & $231(6.0)$ \\
Age at menarche (years) & & $13.6(1.5)$ \\
Menopause & & $2137(55.5)$ \\
Age at menopause (years) & & $47.2(5.8)$ \\
Femoral T score & $-0.93(1.24)$ & $-1.43(1.18)$ \\
Lumbar T score & $-1.40(1.48)$ & $-1.45(1.54)$ \\
\hline
\end{tabular}

* Numbers are presented as mean (standard deviation in parenthesis) for numerical variables and frequency (percentage in parenthesis) for categorical variables.

Table 2: Classification of T scores according to WHO criteria in different sites*

\begin{tabular}{ccccccc}
\hline & Lumbar spine & & Total hip \\
\hline & No. & $\%$ & $\begin{array}{c}95 \% \text { Confidence } \\
\text { Intervals }\end{array}$ & No. & $\begin{array}{c}\% \\
95 \text { Confidence } \\
\text { Intervals }\end{array}$ \\
\hline Osteoporosis (T = -2.5) & 1036 & 24.7 & $23.4-26.0$ & 518 & 12.4 & $11.4-13.4$ \\
Osteopenia $(-\mathbf{2 . 5}<\mathbf{T}=-\mathbf{I})$ & 1605 & 38.3 & $36.8-39.8$ & 1592 & 38.0 & $36.5-39.5$ \\
Normal $(\mathbf{T}-\mathbf{- 1})$ & 1547 & 36.9 & $35.5-38.4$ & 2078 & 49.6 & $48.1-51.1$ \\
\hline
\end{tabular}

Table 3: Distribution of diagnostic discordances according to WHO criteria in different genders*

\begin{tabular}{|c|c|c|c|}
\hline & Male participants $(n=340)$ & Female participants $(n=3848)$ & Total $(n=4 \mid 88)$ \\
\hline Major T-score Discordance & $7(2.1)$ & $108(2.8)$ & I I 5 (2.7) \\
\hline $\begin{array}{c}\text { Hip Osteoporosis, Normal } \\
\text { Lumbar }\end{array}$ & 5 & 16 & 21 \\
\hline $\begin{array}{l}\text { Hip Normal, Lumbar } \\
\text { Osteoporosis }\end{array}$ & 2 & 92 & 94 \\
\hline Minor T-score Discordance & $117(34.4)$ & 1514 (39.3) & |63 I (38.9) \\
\hline $\begin{array}{l}\text { Hip Osteoporosis, Lumbar } \\
\text { Osteopenia }\end{array}$ & 10 & 99 & 109 \\
\hline $\begin{array}{c}\text { Hip Osteopenia, Lumbar } \\
\text { Osteoporosis }\end{array}$ & 39 & 515 & 554 \\
\hline Hip Osteopenia, Normal Lumbar & 35 & 220 & 255 \\
\hline Hip Normal, Lumbar Osteopenia & 33 & 680 & 713 \\
\hline T-score Concordance & $216(63.5)$ & $2226(57.8)$ & $2442(58.3)$ \\
\hline Hip and Lumbar Osteoporosis & 50 & 338 & 388 \\
\hline Hip and Lumbar Osteopenia & 93 & 690 & 783 \\
\hline Hip and Lumbar Normal & 73 & 1198 & $|27|$ \\
\hline
\end{tabular}

\footnotetext{
* Numbers are presented as frequency (percentage in parenthesis).
} 
Table 4: Results of multivariate logistic regression analysis for risk factors of major and minor discordance getting T-score concordance at lumbar and femoral sites as the reference

\begin{tabular}{ccc} 
Variables & Minor Discordance & Major Discordance \\
\hline Gender (female) & & $1.02(0.45-2.3)$ \\
Age decade & $1.09(0.85-1.4)$ & $1.5(1.2-1.9)^{*}$ \\
Age group (>65 years) & $1.2(1.1-1.3)^{*}$ & $1.4(0.70-2.7)$ \\
Corticosteroid use & $1.2(1.01-1.6)^{*}$ & $0.71(0.37-1.3)$ \\
Body Mass Index (>30 kg/cm $\mathbf{2})$ & $0.89(0.73-1.1)$ & $1.7(1.2-2.6)^{*}$ \\
History of osteoporotic fracture & $1.01(0.87-1.2)$ & $1.3(0.29-5.5)$ \\
Smoking & $1.1(0.59-2.0)$ & $0.49(0.12-2.1)$ \\
Menopause & $0.66(0.45-0.97)^{*}$ & $1.7(1.01-2.7)^{*}$ \\
Hormone Replacement Therapy & $1.3(1.1-1.5)^{*}$ & $0.54(0.36-0.82)^{*}$ \\
Age at menarche (> 13 years) & $0.37(0.16-0.82)^{*}$ & $0.82(0.50-1.3)$ \\
Age at menopause (> 50 years) & $1.1(0.90-1.3)$ & $2.0(1.2-3.4)^{*}$
\end{tabular}

* indicates significant odds ratio. Numbers are presented as odds ratio ( $95 \%$ confidence intervals in parentheses).

measurement sites in other 2442 (58.3\%) participants were not different. Distribution and pattern of this variable in different genders is depicted in Table 3.

T-score discordance was more prevalent in women than men $(42.2 \%$ versus $36.5 \%, P=0.042)$. The mean age of participants with discordance (54.8 years) was higher than the other group (52.5 years, $\mathrm{P}<0.001)$. In 3848 female participants, the number of post-menopausal women with diagnostic discordances (951 of 2027) was significantly higher than pre-menopausal participants with discordance ( 671 of $1821 ; \mathrm{P}<0.001)$. In multivariate analysis (Table 4), two genders lost their difference in occurrence of discordance. Effects of age and menopause were established with their significant odds ratios. Participants with late menopause (age at menopause > 50) were more likely to show T-score discordances. Obesity defined as BMI over 30 was recognized as a risk factor for major discordance and smoking as a protective factor against minor discordance. Hormone replacement therapy was a significant protector against both.

\section{Discussion}

This study reveals that, using WHO criteria for definition of osteoporosis and osteopenia, a significant fraction of patients $(41.7 \%$ in this study) would show T-score discordance between hip and spine sites. Most of these discordances $(38.9 \%)$ are from minor category, presenting difference on only one class, and could be due to minor variation in BMD techniques or some minor physiologic dissimilarity. Minor discordance generally does not influence the overall prognosis of patients; however, in the case of patients with one site normal and the other osteopenic, follow up of patients with hip osteopenia seems reasonable [7].
The multivariate analysis we have implemented to the data could aid clinicians and diagnosticians to approach patients with different characteristics. According to our results, BMD measurement in both sites is necessary at least for older patients and post-menopausal women especially those with delay in menopause. Hormone replacement therapy, however, could decrease the diagnostic discordance and patients receiving estrogen and progesterone are more likely to have similar results in DXA scans of lumbar and femoral areas. This could be the result of drug effects on the BMD of lumber area [10].

Generally, five different causes have been proposed for occurrence of discordance [5]. Physiologic discordance is related to the skeleton's natural adaptive reaction to normal external and internal factors and forces. An example of this type of discordance is the difference observed between the dominant and non-dominant total hip. Pathophysiologic discordance is seen secondary to a disease. Common examples include vertebral osteophytosis, vertebral end plate and facet sclerosis, osteochondrosis, and aortic calcification. Anatomic discordance is owing to differences in the composition of bone envelopes tested. An example is the difference in T-scores found for the PA lumbar spine and the supine lateral lumbar spine in the same patient. Artifactual discordance occurs when dense synthetic substances (such as metal from zipper, coin, clip, etc) are within the field of region of interest of the test. And finally, technical discordance occurs when the technician improperly positions the patient for the test or the hardware or software used to acquire the test data is out of order.

Major discordance was observed in $2.7 \%$ of our participants, which is in agreement with the results of similar studies. In both major and minor discordances, lower 
BMD for lumbar spine was more prevalent. This could be due to several reasons. The difference between velocities of bone loss in different parts of human body could be the main reason [11]. Trabecular bones (typical of lumbar area) are known to have a more rapid rate of deprivation in early post-menopausal state in comparison to cortical bone (typical of proximal femur) [12]. Moreover, most of the etiologies of the secondary osteoporosis (such as glucocorticoid excess, hyperthyroidism, malabsorption, liver disease, rheumatoid arthritis, and medications) first affect spinal column [13]. This will lead to higher prevalence of lumbar osteoporosis. In addition, weight bearing is a known cause of physiologic dissimilarity, which can cause rise in bone density especially in the hip and femur regions [14]. This mechanism could be the reason of more major T-score discordances observed by increment of BMI in this study.

In $30 \%$ of our participants, the lumbar T-score was higher than hip T-score and this culminated in poorer hip diagnoses in $9.2 \%$ of participants. This phenomenon could be regarded as 'inverse discordance' and several factors may be involved in its occurrence. One of these reasons is the prevalent vitamin $\mathrm{D}$ deficiency in our participants. A recent nationwide study with random sampling from five major cities in Iran reported a high prevalence (about $80 \%$ ) for vitamin D deficiency in Iranian population [15]. Other studies have confirmed this finding [16,17]. Basic studies have revealed that decrease in serum concentrations of vitamin $\mathrm{D}$ by means of raising serum parathyroid hormone (PTH) would induce reduction in density of cortical bones and may have a supportive role for density of trabecular bones [18]. The other reason for 'inverse discordance' could be due to other diseases such as minor compression fractures in lumbar area, joint sclerosis, and aortic calcification $[19,20]$. These ailments can induce errors in the estimation of lumbar BMD and falsely higher values.

The observation of 'inverse discordance' could not be regarded as a direct influence of more significant bone loss in femoral region. A known phenomenon named 'birth cohort effect' can play a role [21]. This indicates that, in the particular section the data have been gathered, a specific observed finding could not be interpreted for the effects of age and time passing. In this study, the reason for lower femoral BMD can be insufficient bone gain during puberty in this area. Latest findings indicates that peak bone mass of Iranian population are about $5 \%$ lower than that of western population $[22,23]$. Decreased bone density in hip region could lead to start of bone loss from lower amounts in older ages and post-menopausal states. This can lead to femoral osteoporosis without significant decrease in lumbar BMD.
This study, as every other cross-sectional study, has a number of limitations. We could not rule out the possibility of referral bias for this study. As the study was performed in a referral center affiliated to a teaching hospital, the assumption of similarity of study population to exact community is not reasonable and we could not generalize the results to the Iranian population. The other limitation is the choice of multivariate analysis used in this study. With the current analysis, prediction of the presence or absence of T-score discordances is possible. However, prediction of the situation of one site according to results of the other site or choosing one site to measure BMD need further evaluations and analyses which was behind the scope of this study. Future studies using more powerful statistical analyses with larger sample sizes are needed to establish these imperative questions.

The importance of existing discordance on the prognosis and fracture risk of patients needs further prognostic studies with long follow-up designs. The high prevalence of Tscore discordance could induce some problems for the physicians in decision-making regarding these patients. In general, high prevalence of discordance in this study and similar studies suggests some defects in the cut-off values for definition of osteoporosis and osteopenia proposed with the WHO [5]. To eliminate this problem, further studies to re-calculate ranges for definition of these diagnoses (considering diagnostic and therapeutic necessities) seem to be needed.

\section{Conclusion}

In summary, this study indicates that about $40 \%$ of participants evaluated for bone density changes in a referral center may show diagnostic discordance, majority of them from minor class. This phenomenon should be regarded as a real and prevalent finding and physicians should become familiar with this topic. Clinicians should look for possible cause or causes of this occurrence and develop a particular strategy approaching to these patients.

\section{Competing interests}

This study was supported by a grant from Endocrinology \& Metabolism Research Center of Tehran University of Medical Sciences.

\section{Authors' contributions}

In advance, suggestion of the design of the study was from AS. Data extraction and initial analysis were done by NKT and $\mathrm{AH}$. AM performed additional analyses and wrote the first draft of the paper. AS and BL both had helpful and valuable comments in revising the paper. All authors read and approved the final manuscript. 


\section{References}

I. Consensus development conference: Diagnosis, prophylaxis and treatment of osteoporosis. Am J Med 1991, 90:107-10.

2. Consensus development conference: Diagnosis, prophylaxis and treatment of osteoporosis. Am J Med I993, 94:646-50.

3. World Health Organization: Assessment of fracture risk and its application to screening for postmenopausal osteoporosis. In Technical report series 843 Geneva: WHO; 1994.

4. Faulkner KG, von Stetten E, Miller P: Discordance in patient classification using T-scores. J Clin Densitom 1999, 2:343-50.

5. Woodson G: Dual X-ray absorptiometry T-score concordance and discordance between the hip and spine measurement sites. J Clin Densitom 2000, 3:319-24.

6. Mulder JE, Michaeli D, Flaster ER, Siris E: Comparison of bone mineral density of the phalanges, lumbar spine, hip, and forearm for the assessment of osteoporosis in postmenopausal women. J Clin Densitom 2000, 3:373-8I.

7. Abrahamsen B, Stilgren LS, Hermann AP, Tofteng CL, Barenholdt $O$, Vestergaard P, Brot C, Nielsen SP: Discordance between changes in bone mineral density measured at different skeletal sites in perimenopausal women - implications for assessment of bone loss and response to therapy: The Danish Osteoporosis Prevention Study. J Bone Miner Res 2001, 16:1212-9.

8. Hans D, Rizzoli R, Thiebaud D, Lippuner K, Allaoua S, Genton L, Luzuy F, Krieg MA, Jaeger P, Slosman DO: Reference data in a Swiss population. Discordance in patient classification using T-scores among calcaneum, spine, and femur. J Clin Densitom 200I, 4:29l-8.

9. O'Gradaigh D, Debiram I, Love S, Richards HK, Compston JE: A prospective study of discordance in diagnosis of osteoporosis using spine and proximal femur bone densitometry. Osteoporos Int 2003, 14:13-8.

10. Wells G, Tugwell P, Shea B, Guyatt G, Peterson J, Zytaruk N, Robinson V, Henry D, O'Connell D, Cranney A, Osteoporosis Methodology Group and The Osteoporosis Research Advisory Group: Metaanalyses of therapies for postmenopausal osteoporosis. V. Meta-analysis of the efficacy of hormone replacement therapy in treating and preventing osteoporosis in postmenopausal women. Endocr Rev 2002, 23:529-39.

II. Blumsohn A, Eastell R: Age-related factors. In Osteoporosis Etiology, diagnosis, and management Second edition. Edited by: Riggs BL, Melton LJ III. Philadelphia: Lippincott-Raven Publishers; I995:I6I - I82.

12. Eastell R: Treatment of postmenopausal osteoporosis. N Engl J Med 1998, 338:736-46.

13. Aaron JE, Johnson DR, Paxton S, Kanis JA: Secondary osteoporosis and the microanatomy of trabecular bone. Clin Rheumatol 1989:84-8.

14. Kohrt WM, Snead DB, Slatopolsky E, Birge SJ Jr: Additive effects of weight-bearing exercise and estrogen on bone mineral density in older women. J Bone Miner Res 1995, I 0: I 303-I I.

15. Hashemipour S, Larijani B, Pajouhi M, Bastanhagh M, Soltani A, Javadi E, Adibi H, Shafaee A, Baradar Jalili R: Biochemical parameters of bone in different levels of vitamin D deficiency. Iranian South Medical Journal 2002, I:25-I7. (Article in Persian)

16. Hashemipour S, Larijani B, Adibi H, Javadi E, Sedaghat M, Pajouhi M, Soltani A, Shafaei AR, Hamidi Z, Fard AR, Hossein-Nezhad A, Booya $F$ : Vitamin $D$ deficiency and causative factors in the population of Tehran. BMC Public Health 2004, 4:38.

17. Bassir M, Laborie S, Lapillonne A, Claris O, Chappuis MC, Salle BL: Vitamin $D$ deficiency in Iranian mothers and their neonates: a pilot study. Acta Paediatr 2001, 90:577-9.

18. Lips P: Vitamin D deficiency and secondary hyperparathyroidism in the elderly: consequences for bone loss and fractures and therapeutic implications. Endocr Rev 2001, 22:477-50I.

19. Rand T, Seidl G, Kainberger F, Resch A, Hittmair K, Schneider B, Gluer $C C$, Imhof $H$ : Impact of spinal degenerative changes on the evaluation of bone mineral density with dual energy $X$ ray absorptiometry (DXA). Calcif Tissue Int 1997, 60:430-3.

20. Reid IR, Evans MC, Ames R, Wattie DJ: The influence of osteophytes and aortic calcification on spinal mineral density in postmenopausal women. J Clin Endocrinol Metab 1991, 72: I372-4.

21. Szklo M, Nieto FJ: Epidemiology. Beyond the Basics Gaithersburg, Maryland: Aspen Publishers; 2000.

22. Larijani B, Soltani A, Pajouhi M, Bastanhagh M, Mirfezi SZ, Dashti R, Hossein-neghad A: Bone Mineral density variation in 20-69- years-old population of Tehran. Iranian South Medical Journal 2002, I:49-4I. (Article in Persian)

23. Akbarian M, Davachi F, Salim Zadeh A, Shahram F, Gharib Doost F, Tajy A, Pajoohi M, Jamshidi AR: Bone mass density in normal Iranian population. The Journal of Tehran Faculty of Medicine 2002, 4:333-325. (Article in Persian)

\section{Pre-publication history}

The pre-publication history for this paper can be accessed here:

http://www.biomedcentral.com/1472-6823/5/3/prepub
Publish with Biomed Central and every scientist can read your work free of charge

"BioMed Central will be the most significant development for disseminating the results of biomedical research in our lifetime. "

Sir Paul Nurse, Cancer Research UK

Your research papers will be:

- available free of charge to the entire biomedical community

- peer reviewed and published immediately upon acceptance

- cited in PubMed and archived on PubMed Central

- yours - you keep the copyright

Submit your manuscript here:

http://www.biomedcentral.com/info/publishing_adv.asp
BioMedcentral 